\title{
El Instituto de Medicina Legal de La Habana (Cuba)
}

\author{
The Institute of Legal Medicine of the Havana (Cuba)
}

\section{Resumen}

Se describe la evolución sufrida por el Instituto de Medicina Legal de La Habana desde su fundación hace 50 años. Su comienzo fue realmente un simple cambio de nombre, pero gracias al establecimiento de un sistema de formación de recursos humanos se ha logrado que sus profesionales y técnicos, como principal capital de la institución, hayan alcanzado un alto nivel que ha marcado su real desarrollo y ha permitido que puedan responder a las exigencias periciales de cada momento. Este desarrollo no ha estado aparejado a la introducción de modernas tecnologías, debido a las limitaciones económicas en que siempre se ha desenvuelto su labor, por lo que la utilización de las tecnologías disponibles en otros centros ha sido su principal estrategia de trabajo que le ha permitido mantenerse actualizado y tener resultados comparables a los más avanzados a nivel internacional.

Palabras clave: Medicina legal. Organización. Servicios médico-forenses. Habana. Cuba.

\section{Abstract}

The evolution undergone by the Havana Institute for Legal Medicine is described, since its foundation 50 years ago. The beginning of the Institute was characterized by a simple change of name. Later, thanks to the introduction of a program for the formation and training of human resources, the Institute's personnel gained a high level of education and performance which allowed the technicians to accomplish their tasks according to the demands of their expert practice. This evolution was not followed by the acquisition of modern technologies. The economic obstacles always present in the Institute's work have hampered it. To alleviate this situation, the personnel of the Institute have profited from the technologies available in other institutions. This strategy has kept them updated, allowing them to obtain advanced results, comparable with the highest international standards.

Key words: Legal medicine. Organization. Forensic medicine services. Havana. Cuba.

\section{Antecedentes}

Al triunfo revolucionario de 1959 , en una recién construida instalación concebida para morgue judicial y banco de órganos y tejidos de la ciudad, se ubican los servicios de lo que sería el primer Instituto de Medicina Legal de La Habana, Cuba. Durante muchos años fue la única instalación en el país dedicada de forma exclusiva a la actuación médicolegal, hasta el surgimiento posterior de algunos centros provinciales o locales de medicina legal, los que respondieron o se anticiparon a la política de desarrollo de la especialidad hasta el año 2000, concebida por el Ministerio de Salud Pública de Cuba a mediados de la década de los años 80 del siglo pasado.

Su primer director y fundador, el Dr. Genaro Suárez Suárez, médico endocrinólogo de profesión, pero con conocida dedicación y amplios conocimientos para su época de la medicina legal, tuvo una visión acertada de lo que requería el futuro del país, y con el conocimiento que tenía de lo que en el campo de la medicina legal ocurría en el mundo, ayudado por su innegable espíritu emprendedor y poder de decisión, nombró de un día para otro la recién construida instalación con el flamante nombre de Instituto.

\section{J. González ${ }^{1}$ \\ AM. Basanta ${ }^{2}$}

${ }^{1}$ Ex Director del Instituto de Medicina Legal. Rector de la UCM de La Habana, Cuba ${ }^{2}$ Directora del Instituto de Medicina Legal de La Habana, Cuba

Correspondencia: Dr. Jorge González Pérez E-mail: rectorch@infomed.sld.cu
Fecha de recepción: 15.MAR.2010

Fecha de aceptación: 15.MAR.2010 
Pero el cambio de nombre realizado en su fachada no provocaría per se las necesarias transformaciones científicas y conceptuales esperadas para tal denominación; se requerirían, a partir de ese momento, muchos años de trabajo sostenido y desarrollo de proyectos, no todos logrados, que han sido la guía de trabajo para estos últimos 50 años, los que han transcurrido desde ese momento histórico de cambio de nombre hasta la actualidad, en que todavía reconocemos que quedan muchas cosas pendientes de lograr y muchas tareas nuevas que proyectar.

La medicina legal, no reconocida todavía como especialidad 50 años atrás en Cuba, era sólo una dedicación empírica de algunos médicos, la mayor parte de las veces de forma no exclusiva. Sin embargo, esto no impidió poder contar con muchas figuras ilustres en la historia del país en el campo médico-legal, sobre todo provenientes de la Cátedra de la Universidad de La Habana (de la que las carreras de medicina y derecho fueron fundadoras en su concepción como primera Universidad de Cuba en 1728).

Se reconoce como primer maestro de medicina legal en el país al español Don José de Lletor y Castro Verde, quien comenzó a impartir la asignatura en la carrera de medicina en el año 1839, y como primer cubano en hacerlo, a Don Ramón Zambrana y Valdés, que fuera su sucesor. Muchas han sido las figuras que han engrandecido la especialidad en épocas posteriores, por sólo citar a algunas de ellas se destacan los profesores Raimundo de Castro y Bachiller, en la primera mitad del siglo XX, y Francisco Lancís y Sánchez e Ignacio Fournier Ruiz en la segunda mitad de ese siglo.

En el resto del país se destacaron algunos médicos forenses. Era un nombramiento dado por los jueces en su demarcación judicial a aquellos médicos que auxiliaban en la administración de justicia, basado en lo establecido por la Ley de Enjuiciamiento Criminal, sustituida posteriormente por la Ley de Procedimiento Penal.

En todo ese período se subordinó la medicina forense a la administración de justicia, y no es hasta el año 1965 que se transfiere el Cuerpo Médico Forense del Ministerio de Justicia al Ministerio de Salud Pública, lo que incluyó también el llamado Instituto de Medicina Legal que ya funcionaba con ese nombre desde 1960.

Poco después, la salud pública crearía de forma paulatina diferentes institutos de investigaciones vinculados a las especialidades de endocrinología, cardiología y cirugía cardiovascular, nutrición, gastroenterología, angiología, reumatología, entre otros. Tendrían la misión de propiciar el desarrollo de las distintas especialidades dentro del joven sistema nacional de salud creado; pero tal brillante decisión no abarcó a la medicina legal y, por tanto, no tendría en cuenta el pionero centro nombrado con esa categoría desde 1959 en el campo de la medicina legal, que ya había pasado a su subordinación, y que seguiría diferente camino dentro del propio sistema de salud.

\section{Preparación del personal}

La morgue judicial de La Habana, que antes estuvo ubicada en dos recintos diferentes sucesivos (uno independiente en la parte antigua de la ciudad y otro ubicado dentro del Cementerio "Cristóbal Colón" de La Habana, donde hoy radica un área de museo que no incluye ese dato para el visitante), vio como un importante paso en su desarrollo la construcción de la edificación ubicada en un céntrico lugar de la ciudad, en la Avenida Independencia y la calle Hernán Cortés, esta última mutilada en su posterior desarrollo y que sólo se limita hoy a dar acceso a dicha instalación.

La vida motivó, por causas aun no bien conocidas, que el frente de la edificación pasara a ser el fondo de la misma, y que lo que fue concebido para dar espacio a 24 trabajadores y servicios a una ciudad de poco más de un millón de habitantes, sea hoy el centro que responde a todas las actuaciones médicolegales de la capital del país, con más de dos millones de habitantes, y su población flotante (incluido gran número de turistas de diferentes nacionalidades), y que tenga que dar espacio a más de 150 trabajadores, con servicios de 24 horas todos los días del año. También es el centro docente de mayor actividad en el campo de las ciencias forenses en todo el país y por donde pasan estudiantes de medicina, derecho, policiología, entre otros, y donde se desarrollan otras muchas actividades de formación del pregrado y el postgrado.

Pero todo ese incremento de las actividades no ha estado acompañada de la imaginada ampliación ni modernización de sus instalaciones, siendo más lo que se nos pide que lo que se nos da. Esto no ha impedido que se mantenga un alto nivel de respuesta a cada una de las peritaciones que se solicitan, y que los modestos equipos recibidos no sean razón para no realizar otras pruebas de mayor envergadura y complejidad, acorde al desarrollo actual de las ciencias forenses en el mundo.

Como no hay magia en esa respuesta, la estrategia ha sido montada sobre la base de la identificación y utilización de toda la moderna tecnología existente en la ciudad, esté o no dentro del sistema de salud, 
pues por las características de nuestro sistema social se facilita mucho la cooperación libre entre las instituciones, incluso de forma gratuita. Por tales razones hemos podido acceder en todo este tiempo tanto a un microscopio electrónico de barrido, a un cromatógrafo de gases acoplado a un espectrómetro de masas, o a un moderno secuenciador de ADN, entre otros medios, para poder dar la respuesta necesaria a la autoridad que solicita el peritaje.

Entonces ha sido y seguirá siendo la preparación del personal la clave para el trabajo en el Instituto de Medicina Legal de La Habana. Tener profesionales y técnicos capaces de saber qué puede hacerse en cada caso, qué posibilidades ofrece la peritación médico-legal a la actuación judicial, para entonces saber tomar la muestra adecuada, buscar el lugar propicio y la técnica necesaria para dar respuesta al problema que se nos presenta, aun cuando ella no esté disponible en nuestra institución, es la forma de trabajo que inculcamos en nuestros educandos y con la cual hemos tenido éxito.

Para lograr la preparación que exige la vida profesional de hoy, en 1971 se creó en el país el sistema para la formación de especialistas de medicina legal, mediante el programa del régimen de las residencias médicas, que ya ha transitado mediante un sistemático perfeccionamiento por cuatro programas diferentes, cada uno de ellos adaptado a los necesarios cambios que genera la aparición del nuevo conocimiento y el desarrollo de la ciencias en general, a lo que no son ajenas las ciencias forenses.

En un principio se trabajó en un programa de dos años de estudio a tiempo completo, que se realizaba sólo en el Instituto de Medicina Legal de La Habana y que tenía algunas rotaciones en servicios ajenos a la medicina legal (por ejemplo, la traumatología se hacía en servicios de ortopedia), que luego pasó a tres años, con todas las rotaciones vinculadas a la medicina legal y en lo posible dentro del servicio médico-legal correspondiente, con la participación de los servicios y especialistas ya formados en determinadas partes del país.

Después, al ya tener en todo el país especialistas formados en el campo médico-legal, con servicios y laboratorios que apoyan la labor pericial, se autorizó la formación de los nuevos especialistas en la mayor parte de las provincias del país, previa acreditación del escenario y la categorización de sus profesores; esto no es del todo homogéneo, y mientras existen provincias donde se puede desarrollar toda la residencia sin dificultad, existen otras que deben enviar sus residentes a otro lugar para poder realizar estancias o rotaciones que no tienen acreditadas en la misma, como por ejemplo en toxicología o biología forense.

En el plan de la residencia, que es a tiempo completo durante esos tres años, tal y como ocurre con la práctica de los especialistas forenses que es a tiempo completo dentro de la profesión en todo el país, se establecen un número mínimo de actuaciones en diferentes campos para poder considerar como aceptable su estancia o rotación. Esto motiva que en ocasiones, aunque su servicio tanatológico esté acreditado para la docencia, si al concluir el tiempo establecido, digamos dos meses, el residente no ha cumplido el número mínimo de casos, por ejemplo la necropsia del estrangulado, debe ir a otro servicio donde el número de casos sea mayor para poder cumplir con la norma de trabajo establecida como requisito para considerar su rotación como válida.

En todos los casos, como está establecido en el país para todas las especialidades que se caracterizan por la formación desde los servicios, a tiempo completo y por la que se recibe un salario, el residente debe aprobar el examen práctico de cada rotación antes de ir a su examen teórico. Además de la evaluación de las rotaciones o estancias, debe existir un examen de pase de cada año y uno final (también llamado "estatal"), que sólo tienen capacidad para realizarlo los Tribunales nombrados por el Ministerio de Salud Pública de forma centralizada, que exigen también la presentación de una investigación científica en el campo de la especialidad como obligación para poder presentarse a los exámenes prácticos y teóricos ya mencionados.

\section{La práctica forense básica}

Desde su concepción original, el Instituto de Medicina Legal se han caracterizado por el desarrollo de las actuaciones médico-legales básicas, entiéndase las investigaciones tanatológicas y de la clínica médico-legal, complementadas siempre por los estudios de laboratorios necesarios.

Por tales razones, en el Instituto se realizan diariamente los reconocimientos o las necropsias de los casos que son remitidos por la autoridad competente a dicha instalación: en principio todas las muertes violentas, a las que se suman todos los casos de extranjeros y sujetos que en el momento de su muerte tuvieran afectado por alguna causa su régimen de libertad personal (aunque no se trate de una muerte violenta), los casos de muerte sospechosa de criminalidad, los fallecidos desconocidos y otras muertes que por sus características sugieran una actuación 
médico-legal, lo que hoy promedia unos 1.500 fallecidos por año que ingresan en el Instituto.

En el último período, con la inauguración en el 2007 del crematorio de cadáveres de la Ciudad de La Habana, han aumentado los ingresos por reconocimiento al ser necesaria una actuación médico-legal en dichos cuerpos antes de la cremación, con la práctica de la necropsia en algunos casos en que ésta no había sido realizada y el perito lo consideró oportuno; se realizan entre 8 y 10 incineraciones diarias, todas las cuales exigen el examen médicolegal previo con la expedición de la boleta de autorización de cremación por la guardia de medicina legal.

A dicho centro se remiten todos los fallecidos de muerte violenta que ocurren en la Ciudad de La Habana, que tiene una extensión de $725 \mathrm{~km}$ cuadrados y está dividida en 15 municipios, con una población de 2,2 millones de habitantes. También se remiten de forma excepcional algunos casos de muerte violenta ocurridos en otras localidades del país. Por ejemplo, atendiendo a la resolución del Ministro de Salud Pública que regula el trabajo médico-legal con fallecidos extranjeros desde 1990, en la cual se dispone la metodología para la realización de la necropsia y la toma de muestras para estudios complementarios, desde los histopatológicos y microbiológicos, hasta los toxicológicos y de otro tipo, cuando en algún lugar del país no se puedan cumplir las exigencias de dicha norma, deberá ser remitido el cadáver al lugar donde existan dichas condiciones, por lo que muchos casos son remitidos al Instituto de Medicina Legal, atendiendo además a que, de todas formas, la salida del cuerpo se va a realizar desde la capital del país, por ser este el lugar donde radican los Consulados de todas las embajadas acreditadas, lo que facilita el trámite de repatriación.

En el Instituto se realiza una guardia física de 24 horas que cuenta con 2-4 médicos por turno, los que son acompañados por 2 o 3 auxiliares para la práctica de las necropsias y otras actuaciones médicolegales, y cuenta también con un vehículo de guardia tipo furgoneta en el cual se concurre al lugar del hecho y donde se traslada también el cuerpo del fallecido de retorno a la instalación para continuar la labor médico-legal que corresponda. Existe también un personal de apoyo institucional, que incluye el personal de recepción y custodia, así como auxiliares generales para mantener la limpieza del centro, y otros localizables, como el que realiza la labor de filmaciones o las pruebas de laboratorios cuando son de urgencia.

De lo anterior se desprende que una de las actuaciones tanatológicas básicas, y que consideramos de gran importancia en nuestra labor pericial, es la de concurrir al lugar del hecho y realizar el levantamiento de cadáveres, que significa el conocimiento de la actuación desde el propio lugar de la muerte o donde apareció el cuerpo hasta la realización de la necropsia médicolegal, lo que siempre se hace por más de un perito.

Es práctica habitual en nuestra institución la discusión colectiva de los casos diariamente al concluir el turno de guardia, lo que se hace en el propio salón de tanatología a las 8:30 AM, con la participación de los médicos que estuvieron de guardia y realizaron las actuaciones, y el resto de los legistas y patólogos de la institución. El actuante expone las piezas anatómicas disecadas y explica la descripción de las mismas, dando a conocer los antecedentes del caso y los datos del levantamiento, si se hizo, que se visualiza mediante video si existe y si éste fuese de interés para la mejor comprensión de caso en estudio, significando en forma resumida lo encontrado en cada caso.

Acto seguido, el Jefe del Departamento de Tanatología somete a la consideración de todos los presentes la información ofrecida, que luego de una fase de preguntas para aclarar o profundizar en un dato concreto, pasa entonces a la fase de comentarios o de las interpretaciones de cada cual sobre lo ocurrido, comenzando con el personal en formación de menor rango, alumnos ayudantes y residentes de años inferiores hasta los de años superiores, para culminar con los especialistas y profesores presentes, atendiendo también a la categoría de los mismos, para concluir con las palabras de la Directora del Instituto, quien orienta entonces la labor a realizar en el caso, respetando los criterios individuales de cada perito, pero sin que ello ponga en riesgo o comprometa la responsabilidad institucional para seguir el mejor curso posible de la investigación, lo que hace que siempre prime el criterio colectivo sobre el individual.

Como actuaciones que también se realizan en el campo tanatológico están las exhumaciones de cadáveres y restos óseos, y su investigación, tanto antropológica, biológica, odontológica, como médico-legal en general, que permite la identificación y explicación sobre las posibles causas de la muerte y las circunstancias en las cuales se produjo; para esto los médicos se apoyan en la presencia de dos antropólogos entre los profesionales que laboran a tiempo completo dentro del Instituto.

La clínica médico-legal se realiza durante las 24 horas del día por el mismo personal de guardia para atender las urgencias que en este campo puedan existir, al tiempo que se organiza sistemáticamente la consulta médico-legal en toda la ciudad, por zonas, para atender los casos que requieran de este servicio, sobre 
todo el atestado de sanidad de lesionados, cuyo monto es de más de 12.000 actuaciones por año.

El resto de las actuaciones de la clínica médico-legal no llega a 1.000 por año, por ser muy baja la cifra de delitos sexuales y problemas con droga existente en la ciudad, y también muy poco frecuente la peritación para conocer el estado mental del sujeto al momento del hecho, así como excepcional la determinación de edad y otras peritaciones en vivos.

Si bien es muy frecuente la determinación del estado alcohólico en sujetos vivos, sobre todo en relación con el tránsito, dicho examen clínico se realiza por los diferentes servicios asistenciales de la ciudad ajenos a la medicina legal, que funcionan durante las 24 horas y pertenecen al sistema de salud, y sólo muy excepcionalmente son llevados al Instituto sujetos para realizar tal determinación clínica. Sin embargo, sí se reciben diariamente muestras biológicas de muchos de estos sujetos para ser procesadas por nuestro laboratorio toxicológico.

En ocasiones, la guardia médica es llamada para concurrir a algún centro de detención policial para examinar un sujeto desde el punto de vista clínico o mental, o para los mismos fines se concurre a centros asistenciales donde el sujeto está ingresado y su examen resulta fundamental, lo mismo para evaluar la gravedad real de sus lesiones, por considerar la autoridad que la clasificación y el pronóstico emitido por el médico de asistencia no es el correcto, que para establecer cuál es el posible orificio de entrada y salida en casos de disparo por arma de fuego donde el médico de asistencia no ha demostrado de forma sólida sus conocimientos sobre dicha interpretación, entre otras causas de peritación extrainstitucional.

\section{Solución a las necesidades de los la- boratorios y del desarrollo}

Las ciencias forenses y en particular la medicina legal, por regla, no han sido asuntos priorizados en la mayoría de los países del mundo, peor aun en momentos de crisis económica, de recortes y reajustes, con limitantes de presupuestos y la existencia de otras prioridades, aun en el mundo desarrollado, de lo que ya dejábamos constancia en el año 2000 en la denominada "Acta de Varadero" (Anexo 1), con la cual concluimos el Congreso Internacional de Ciencias Forenses celebrado en dicho balneario en ese año.

Entonces la solución a nuestras necesidades durante este medio siglo ha tenido que basarse, como antes mencionamos, en la estrategia de búsqueda de oportunidades para mantener un desarrollo científico de alto nivel sin disponer dentro de nuestro Instituto de la alta tecnología propia de dicho desarrollo, pero sobre la base de la alta calificación de su personal en la indicación, realización e interpretación de las posibles pruebas a practicar según las tendencias modernas de la peritación en ciencias forenses en el mundo.

Ejemplo de esta estrategia, propia para países en desarrollo pero que hoy no excluye por la crisis a los desarrollados, lo ha sido, en nuestro Instituto, el laboratorio de toxicología forense. El mismo fue concebido desde su fundación en el año 1959, tuvo incluso sus primeros trabajadores desde esa época, luego buscaron otras opciones de trabajo pues no se pudieron concretar sus esfuerzos hasta finales de la década de los 70 del siglo pasado, lográndose su real puesta en marcha dentro del Instituto en 1980 , con un equipamiento mínimo.

En todos estos años, a pesar de eso, se han realizado miles de investigaciones y se ha resuelto un sin número de problemas en el campo toxicológico forense, desde las investigaciones de barbitúricos en los años de su uso sostenido, apoyados por los laboratorios de la industria farmacéutica, luego de los plaguicidas, donde buscamos el concurso de los prestigiosos laboratorios de la agricultura, hasta la búsqueda de múltiples sustancias empleando los recursos de la red de laboratorios de criminalística del Ministerio del Interior o el Laboratorio Antidoping del organismo deportivo; dando respuesta en lo fundamental a la peritación toxicológica como lo exige la medicina legal de hoy, usando la tecnología existente en el país, y contando con verdaderos expertos en cada campo, los que nos han permitido elevar el nivel de nuestros peritos en dicho trabajo conjunto.

Eso no niega que trabajemos el control de calidad propio y que estemos vinculados a instituciones internacionales que así lo verifican, pues el uso de uno u otro equipo o la realización de la prueba en uno u otro lugar, no limita que se sigan las reglas establecidas para la peritación forense, con la cadena de custodia de las muestras y toda la seguridad y confidencialidad con que debe trabajarse en nuestra peritación, lo que es una exigencia permanente en la labor que cada día realizamos.

Similar estrategia se ha seguido en otras peritaciones, como por ejemplo la investigación de ADN cuyo laboratorio propio inauguramos en 1995, pero desde mucho antes establecimos peritos nuestros tanto en el Laboratorio Central de Criminalística como en el Laboratorio de Genética del Hospital Clínico Quirúrgico "Hermanos Ameijeiras" y el Instituto de Hematología 
e Inmunología, centros de alto desarrollo en el sistema de salud y con los cuales hemos trabajado de forma estrecha, al igual que con otros tantos de la ciudad, y con los que tenemos como norma seguir trabajando.

\section{Regulaciones y procedimientos para el trabajo médico-legal}

Existen en el Instituto de Medicina Legal de La Habana un conjunto de documentos que establecen los procedimientos para las actuaciones que se deben realizar en cada caso, lo que se elabora por vez primera a finales de la década de los años 70 del pasado siglo y se han actualizado con relativa sistematicidad.

Éstos recogen una información preliminar de la actuación, el motivo de ella, las bases legales para la misma, el lugar de la actuación, el número de peritos que deben estar presentes, la manera de informar y si existen modelos oficiales específicos para la misma, las muestras que deben ser recogidas cuando ello procede, así como los instrumentos o materiales que se necesitan para poder realizar dicha peritación, entre otros detalles prácticos.

Aunque la Ley deja abierta a la autoridad aceptar quiénes son peritos médicos, en la práctica sólo los dedicados a tiempo completo a la medicina legal son los que realizan las principales actuaciones médicolegales, que siempre se realizan por solicitud de la autoridad competente, que en Cuba son los instructores judiciales, los fiscales y los jueces, que actúan a tres niveles jerárquicos: municipal, provincial o nacional.

Es, por tanto, el Instituto de Medicina Legal de La Habana el centro de mayor actividad pericial en el campo médico en el país, para lo cual sólo dispone de unos 30 médicos en su planta, muchos de los cuales cumplen otras tareas fuera del país o en otros servicios de forma provisional.

Por acuerdo conjunto entre el Ministerio del Interior, el Ministerio de Salud Pública, el Tribunal Supremo y la Fiscalía General de la República, de fecha 3 de mayo, así como la Instrucción No. 110 de 1983 del Consejo de Gobierno del Tribunal Supremo, del 9 de julio de 1983 , se planteó la necesidad de que para investigar los hechos que dieran lugar a denuncias sobre delitos de homicidio o lesiones por imprudencia, presuntamente cometidos por médicos o personal profesional y auxiliar especializado en la rama de la medicina en el ejercicio de su profesión, se dispusiera que en las acciones de instrucción obrara el resultado de la investigación científica realizada por la Comisión para el análisis de la responsabilidad médica.
En el establecimiento de esta normativa ha jugado un papel muy importante el Instituto de Medicina Legal y, durante mucho tiempo, su director fue el Presidente de la Comisión para los procesos de responsabilidad seguidos en Ciudad de La Habana, sobre lo que ya existe una resolución que establece tales procederes, que fue elaborada tomando la experiencia de dicho centro.

El Instituto de Medicina Legal fue el promotor de establecer la metodología para la peritación médico-legal del estado de salud para conocer la compatibilidad de un sujeto con el régimen penitenciario, vigente hoy en el país, así como también la metodología para el funcionamiento de las comisiones provinciales de peritación psiquiátrica forense en el sistema nacional de salud, todo lo que ha favorecido la uniformidad de la peritación forense a lo largo de todo el país.

\section{Colaboración y relaciones internacionales}

Mantenemos buenos vínculos de trabajo con muchas instituciones similares del mundo y con organizaciones que se vinculan con la salud y el trabajo en las ciencias forenses. En los primeros años de su creación se mantuvieron estrechas relaciones con las instituciones homólogas pertenecientes al entonces campo socialista, razón por la cual se recibieron visitas de expertos polacos, húngaros, alemanes, rusos, entre otros.

De igual forma se visitaron esos países, se asistió a numerosos congresos y eventos, y los primeros cursos internacionales se recibieron en dichas instituciones, incluida la primera graduación, en 1987, de un Doctor en Ciencias Médicas en el campo médicolegal, el Dr. Jorge González Pérez que defendió su título en Alemania.

A partir de la década de los 80 se iniciaron importantes relaciones con países de América Latina y fue un eslabón incuestionable para dicho proceso la realización en 1980 de un Congreso Internacional de la especialidad en Cuba, el cual fue presidido por el entonces director del Instituto de Medicina Legal, Dr. Francisco Ponce Zerquera, que también fue el fundador del Grupo Nacional de Medicina Legal en 1971, órgano asesor del Ministro de Salud Pública.

Desde entonces se han organizado eventos internacionales de las ciencias forenses en Cuba, que con el nombre de "Forense", han tenido lugar en los años 1984, 1989, 1993, 1997, 2000, 2003 у 2006, todos bajo el auspicio de la Sociedad Cubana de Medicina Legal, fundada en 1978 por el ilustre Prof. Dr. Francisco Lancís y Sánchez, quien a la edad de 87 
años falleciera en 1993. En todos estos eventos internacionales y en otros muchos con carácter nacional o local, ha jugado un papel protagónico el Instituto de Medicina Legal, que es la imagen real de la medicina legal en el país, cuya directora desde 2001 es la Dra. Alicia Marlenne Basanta Montesinos.

En estos eventos hemos podido contar con la presencia de importantes personalidades de las ciencias forenses del mundo, en particular de la medicina legal, los cuales han visitado el Instituto de Medicina Legal, así como otros que por diversas razones nos han visitado, entre los que podemos citar a los profesores Juan A. Gisbert Calabuig y Joan Carol (España), Ramón Fernández Pérez, Roberto Tocavén y Aurelio Núñez Sálas (México), Gerhard Dietz, Wolfgang Dürwald y R.K. Müller (Alemania), P. Gromov (Rusia), Eduardo Vargas Alvarado (Costa Rica), Luís Alberto Kvitko (Argentina), Cesar Augusto Giraldo (Colombia), Luís Vásconez Suárez (Ecuador), Rolando Costa Ardúz (Bolivia), entre otros.

Se ha participado en reuniones internacionales de directores de Institutos de Medicina Legal organizadas por la Cruz Roja Internacional y la Organización Panamericana de la Salud, así como en otras tantas reuniones internacionales, sobre todo en el tema de desastres, donde se ha tenido una intensa labor en tema de preparativos. El Instituto ha participado en eventos internacionales en la gran mayoría de los países de América Latina, así como ha colaborado en el desarrollo de maestrías en Bolivia y diplomados en Honduras, así como labores asistenciales médicolegales en Bolivia, Haití, Nicaragua, Venezuela, República Dominicana, Ecuador, Angola, Etiopía, Mozambique, Congo y Sudáfrica.

\section{Una experiencia importante}

Su participación en la búsqueda e identificación de enterramientos humanos, unida a la realizada en los preparativos para el manejo masivo de víctimas fatales en situaciones de desastres, han llevado al Instituto de Medicina Legal de La Habana a ser reconocido internacionalmente.

Es bien sabida la participación de dicha institución en la integración de un grupo multidisciplinario que, a partir de 1995 y durante más de cinco años, realizó la búsqueda e identificación en tierras bolivianas de 31 integrantes de la guerrilla de 1967 del Comandante Ernesto Che Guevara. El diseño de un proyecto científico propio, con el uso de novedosas técnicas geológicas y geofísicas, así como computacionales y de teledetección, unido a los tradicionales métodos arqueológicos y antropológicos, dieron a este grupo de científicos la posibilidad de encontrar enterramientos realizados en total secreto 30 años antes.

Esta labor fue precedida por un intenso trabajo realizado a finales de los años 80 por dicha institución, que permitió regresar a Cuba los restos de más de 2.000 cubanos fallecidos cuando cumplían misión internacionalista en diferentes partes del mundo, sobre todo en tierras africanas; significó meses de trabajo para realizar la ubicación, exhumación, identificación, y preparación de cada uno de estos cadáveres u osamentas, todo lo que permitió cumplir con éxito la Ilamada "Operación Tributo".

Desde 1988 ha sido el Instituto de Medicina Legal de La Habana un referente en la elaboración de manuales para el manejo masivo de cadáveres en situaciones de desastres, que ha ido perfeccionando y son hoy parte de las publicaciones especializadas que ha realizado sobre el tema la Organización Panamericana de la Salud.

\section{A manera de conclusión}

La principal conclusión a que podemos arribar es que no siempre es la tecnología lo que determina el desarrollo, pues aun en ausencia de ésta se puede aspirar a metas superiores. La disponibilidad de la misma, bien sea propia o eventual, necesita siempre de la persona preparada y capaz de usarla o al menos saber las potencialidades de la misma para los fines que se desean; por eso, la formación y superación de los profesionales que laboran en el campo forense debe ser la meta superior que nos propongamos, lo que ha sido la estrategia de nuestro Instituto de Medicina Legal.

Resulta evidente que los planteamientos realizados en el año 2000 sobre la situación de la medicina legal en América Latina se mantienen vigentes, o en muchos casos la situación es peor, por lo que debemos seguir luchando para aspirar a tener instituciones médico-legales dignas del desarrollo científico del siglo XXI. 


\section{Bibliografía}

1. Acuerdo conjunto del Ministerio del Interior, el Ministerio de Salud Pública, el Tribunal Supremo Popular y la Fiscalía General de la República, de Cuba de 3 de mayo de 1983.

2. Instrucción No. 110 del Consejo de Gobierno del Tribunal Supremo de la República de Cuba, de 9 de julio de 1983.

3. Lancís y Sánchez F. Lecciones de Medicina Legal. La Habana: Imprenta Universitaria "André Voisin" 1962.
4. Lancís y Sánchez F, et al. Medicina legal. La Habana: Editorial Pueblo y Educación 1999.

5. Ley No. 4 de 23 de noviembre de 1950. Creó el Cuerpo Médico Forense Nacional.

6. Ley No. 1174 de 1965. Pasó el Cuerpo Médico Forense al Ministerio de Salud Pública.

7. Ley No. 5 de 13 de agosto de 1977. Ley de Procedimiento Penal.

8. Ponce Zerquera F. Comunicación personal.

\section{Anexo 1.}

\section{"Acta de Varadero" sobre la situación de la medicina legal en América Latina en el año 2000}

Los Directores de Instituciones médico-legales, los Jefes de Departamentos Docentes y Cátedras de Medicina Legal, los Presidentes de Sociedades y Asociaciones científicas de la rama médico-legal, y demás profesionales Iberoamericanos y de otras regiones del mundo reunidos en el Congreso Internacional de las Ciencias Forenses que ha sesionado del 11 al 14 de octubre del 2000 en el balneario de Varadero, provincia de Matanzas, en la República de Cuba, donde se ha debatido sobre los principales problemas que aquejan a las ciencias forenses, y en particular la medicina legal, en la culminación del Siglo XX en Iberoamérica, con especial énfasis en América Latina, quieren dejar constancia de sus principales problemas, al tiempo de pronunciarse sobre las necesidades y objetivos a cubrir y cumplir en el nuevo milenio, lo que dejamos plasmado en forma resumida en este documento que en lo adelante se denomina "Acta de Varadero".

En América Latina y el Caribe la violencia tiene actualmente características endémicas, sólo en 1993 se registraron en la región 1250 muertes diarias, como promedio, debidas a la violencia, siendo ésta la principal causa de muerte en muchos países, y uno de los motivos de ingreso y atención médica más frecuente.

La pobreza, el desempleo, la desigualdad manifiesta y creciente, la crisis económica reinante y las medidas para enfrentarla, así como una mezcla de factores políticos, sociales y económicos, unidos al consumo de alcohol y drogas como aspectos más significativos, hacen factible un crecimiento en espiral de la violencia al cual los especialistas de las ciencias forenses de la región tenemos que enfrentarnos a diario.
El desarrollo de nuevas tecnologías, puestas indiscriminadamente al servicio del crimen, en desigual proporción a las que se ponen en función de enfrentarlo, hacen cada vez más difícil nuestro trabajo. Por otra parte, los avances tecnológicos en materia vehicular, si bien favorecen el transporte, no están siempre aparejados en nuestra región con similar desarrollo de la red vial por donde se ha de transitar, lo que a la larga se convierte también en un incremento de la violencia.

Aunque pueden existir diferencias notables entre algunos países, señalamos los aspectos más negativos que predominan aun en muchos de ellos, sin referirnos a ninguno en particular, proponiendo así a través de este documento a los Gobiernos de la región considerar su situación particular, instándolos a enfrentarla en la medida de sus realidades y posibilidades.

El estado actual para Iberoamérica puede resumirse de forma más significativa como sigue:

- En la mayoría de los países no existe una atención sistemática ni proyección para el desarrollo de las ciencias forenses, en particular la medicina legal, la que por el contrario ha ido quedando relegada históricamente, más aun debido a la crisis económica en que nos encontramos al final del Siglo que termina.

- La política neoliberal, que lleva implícita las privatizaciones de gran parte de los servicios públicos, afecta medularmente el desarrollo de las ciencias forenses, actividad pública por esencia, lo que provoca una tendencia a la existencia de peritaciones y laboratorios privados que muy pocos ciudadanos pueden pagar y por ende usar, lo que es un acto más de su injusticia.

- La falta de recursos gubernamentales asignados en algunos países a la labor pericial forense impide investigar por igual todos los hechos, lo que motiva que sólo se empleen los existentes 
con un sentido favoritista, generalmente para beneficiar a los que gozan del poder, tanto político como económico.

- Con algunas excepciones, existe una tendencia a la politización de las estructuras administrativas que dirigen las instituciones forenses, las que casi siempre responden a compromisos políticos del Gobierno en el poder, no siempre coincidiendo la designación de los Jefes con la capacidad y proyección científica de los especialistas más destacados, lo que puede traer por consecuencia mediocridad, estancamiento y falta de sistematicidad y continuidad de las políticas de desarrollo en el campo de las ciencias forenses, empobrecidas ya de por si por otros factores.

- A pesar de la existencia de un sin número de Sociedades, Asociaciones, Instituciones, Fundaciones, y de la realización de eventos científicos, relativamente periódicos, en el campo de las ciencias forenses en la región, así como de las facilidades de las comunicaciones modernas, consideramos que existe falta de integración, unidad y cooperación entre los países del área para enfrentar los problemas comunes que nos azotan.

- Debe tomarse como una experiencia positiva los avances logrados en el último período en el tema de las drogas, propiciado fundamentalmente por organismos internacionales, para así ampliarlo a otras esferas de las ciencias forenses que demandan igual tratamiento.

- Ajeno en la mayoría de los casos a las políticas gubernamentales, existen limitaciones creadas por el "caudillismo", la proliferación de "academias", y el divorcio existente entre las Universidades y las instituciones oficiales que realizan las peritaciones forenses, lo que provoca una desvinculación viciosa y mantenida entre la teoría y la práctica, con consecuencias catastróficas.

- Hay ausencia en la mayoría de los países de programas de formación de especialistas; en otros los existentes resultan insuficientes, sobre todo por la falta de recursos y actividad práctica, y no se ha buscado un consenso entre los más avanzados de la región para proponer un "programa tipo", ni la forma en que estos pudieran contribuir en forma altruista al desarrollo de los demás.

- Los profesionales que se dedican en la actualidad a la labor forense oficial reciben general- mente salarios irrisorios y francamente discriminatorios, lo que les obliga a buscar otras fuentes de financiamiento para poder subsistir, eso impide tener una dedicación total a la especialidad, favorece la injusta peritación privada ya mencionada y propicia la corrupción.

- La bibliografía existente en la región es insuficiente; la proveniente de otras regiones no coincide siempre con los enfoques y legislaciones nuestras, pero sobre todo, en muchos casos sus precios la hacen inaccesible, y no siempre las instituciones las adquieren o se la subsidian a los especialistas; esto provoca una insuficiente información científica de los peritos, lo que pudiera convertirse en un futuro en algo grave.

- Los problemas existentes para la instrucción y el perfeccionamiento de los especialistas se ven aun más agudizados por tratarse de la educación permanente, pues pocos son los países de la región que evalúan su competencia y necesidades, ni tienen programada la superación de estos en forma sistemática, al tiempo que no siempre se ofrecen las facilidades a los mismos para la asistencia a eventos, cursos, seminarios, y otras actividades de superación.

- Los continuos cambios legislativos, muchos de ellos justificados por la aparición de nuevas condiciones jurídicas, como pudiera ser el caso de la manipulación genética u otras tecnologías modernas, en ocasiones responden más al rechazo de un nuevo Gobierno por lo realizado por el saliente, lo que a la larga crea inestabilidad de la legislación y en la labor pericial.

- Si bien pudiera interpretarse como parte del normal desarrollo y evolución de las ciencias el desmembramiento científico que se ha venido produciendo en la medicina legal, no es menos cierto que cada día se alejan más la evaluación criminológica en un homicidio de la práctica de la autopsia de la víctima, el estudio de las causas y condiciones de los accidentes del tránsito de la traumatología forense, el diagnóstico de una intoxicación de su investigación epidemiológica y prevención, entre otros, no respetándose así la unidad dialéctica de las mismas.

- La falta de uniformidad o variable subordinación de la medicina legal en cada país de la región, o incluso dentro del propio país, siendo unas veces a las Cortes de Justicia, otras a la Fiscalía General, Ministerio Público o Procuradurías, en algunos países dentro de la Policía Técnica Judicial, Policía Civil o Policía Científica, y hasta incluso ubicarla dentro del Sistema 
de Salud Pública, por sólo mencionar las más frecuentes, crea serios problemas de intercambio, comunicación y colaboración oficial entre las estructuras existentes en los distintos países.

- La no participación del médico legista en el trabajo en el lugar del hecho, o la división arbitraria por etapas de la peritación, que conlleva a que no coincidan los especialistas que laboran en el lugar con los que luego practican la necropsia, ocurre aun en muchos países, lo que se convierte en un elemento organizativo que desorganiza y conspira con la calidad e integralidad de la labor pericial forense.

- La no existencia de Institutos de Medicina Legal en muchos países o regiones de estos, la insuficiente estructura y recursos de los existentes, sin que se modifiquen aquellos que fueron concebidos para un área y población hasta diez o veinte veces inferior a la que hoy deben darle cobertura, y la no existencia de planes de nuevas construcciones, o al menos remodelación o ampliación de los existentes, augura un futuro incierto y funesto a la especialidad.

- La falta de laboratorios forenses, o lo muy limitado de los presupuestos de los existentes, impide en ocasiones llevar a cabo la investigación pericial con el profundo sentido y rigor científico que ella merece, por lo que comprobaciones tan elementales como la presencia de semen en una violación pueden quedar en suspenso y con ello impedir la justa sanción de un culpable. 